\title{
Patterns of infections in older patients acutely admitted to medical wards: data from the REPOSI register
}

\author{
Raffaella Rossio ${ }^{1}$. Ilaria Ardoino ${ }^{2}$. Carlotta Franchi ${ }^{2}$ - Alessandro Nobili ${ }^{2}$ Pier Mannuccio Mannucci ${ }^{3}$. \\ Flora Peyvandi ${ }^{4,5} \cdot$ REPOSI Investigators
}

Received: 6 March 2019 / Accepted: 13 August 2019 / Published online: 23 August 2019

(c) Società Italiana di Medicina Interna (SIMI) 2019

Keywords Older people $\cdot$ Infections $\cdot$ Hospitalizations $\cdot$ Comorbidities

Dear Editor,

In older adults infections are among the leading causes of emergency department visits, hospitalization, morbidity and mortality [1-3]. Infections also occur as adverse events during hospitalization, as highlighted by the large use of antibiotics in this setting, resulting in an increase of hospitalization length and mortality rate [4-6]. There is a paucity of studies, especially in European countries, that did offer a general pattern on all the types of infections occurring in acutely hospitalized older patients, being the literature mainly focused on single type of infections (i.e. pneumonia and urinary tract infections). To fill this gap of knowledge, we chose to observe and describe the prevalence and types

The members of REPOSI Investigators are listed in

"Acknowledgement" section.

Electronic supplementary material The online version of this article (https://doi.org/10.1007/s11739-019-02178-1) contains supplementary material, which is available to authorized users.

Flora Peyvandi

flora.peyvandi@unimi.it

1 Fondazione IRCCS Ca' Granda Ospedale Maggiore Policlinico UOC Medicina Generale - Emostasi E Trombosi, Milan, Italy

2 Department of Neuroscience, IRCCS, Istituto Di Ricerche Farmacologiche "Mario Negri", Milan, Italy

3 Scientific Direction, Fondazione IRCCS Ca' Granda Ospedale Maggiore Policlinico, Milan, Italy

4 Department of Pathophysiology and Transplantation, Università Degli Studi Di Milano, Milan, Italy

5 Angelo Bianchi Bonomi Hemophilia and Thrombosis Center and Fondazione Luigi Villa, Fondazione IRCCS Ca' Granda Ospedale Maggiore Policlinico, Via Pace, 9, 20122 Milan, Italy of infections in a large cohort of older hospitalized patients in the frame of REPOSI (REgistro POliterapie SIMI) register. REPOSI is a collaborative register that involves a large number of Italian internal medicine and geriatric wards. Briefly, patients aged 65 years or more acutely hospitalized during four index periods lasting one week in each season were enrolled and signed an informed consent. The attending physicians were required to compile for each patient a web-based Case Report Form, including the main sociodemographic data, specific diagnoses and their severity according to the Cumulative Illness Rating Scale (CIRS), functional status at hospital admission (measured by Barthel index [BI]) [7] and drug therapies during the whole hospitalization period. This study was approved by the Ethical Committees of all participating hospitals. More details on REPOSI were provided elsewhere $[5,6]$.

Patients recruited in the register in 2008, 2010, 2012, 2014 and 2016 were considered for the purpose of this study. Infections were considered when they were the reason for hospitalization, when reported in the CIRS at admission and also when they occurred during the hospital stay, whether reported as a relevant adverse event or as the indication for antimicrobial therapy. Infections were categorized using the International Classification of Diseases-Ninth Edition (ICD9) according to the physiological system of origin, on the basis of a classification provided in other studies (Supplementary Table 1) [8]. Data were summarized as prevalence $(\%)$, or means and standard deviations when pertinent. The 95\% confidence intervals (CI) were provided as well. The analysis was performed using the SAS/STAT software Version 9.2 (SAS Institute Inc., Cary, NC, USA).

Among the 6047 patients enrolled in the REPOSI register, $2991(49.5 \%, 95 \%$ CI 48.2-50.7\%) were diagnosed with at least one infection, accounting for a total of 3554 infections. Overall 2522 patients (84.3\%) had a single infection, 
$392(13.1 \%)$ two and $77(2.6 \%)$ three or more infections. Almost all the infections had a bacterial or suspected bacterial etiology, but 284 (8.0\%) were viral, mainly hepatobiliary $(N=223)$ and lower-respiratory track $(N=41)$ infections. Socio-demographic and clinical characteristics of the patients with and without infections are reported in Table 1. A total of 1554 patients (52.0\%) were females and their mean age was 80.0 years $(\mathrm{SD}=7.5)$. Compared to patients without infections, almost the same prevalence of females was observed but they were slightly older, the mean age of patients without infections being 78.6 years $(S D=7.4)$ and they took more medications at hospital admission. While the Barthel index (BI) was not available in the 2008 REPOSI run, in the remaining years patients with infections were often functionally impaired, those with a severe dependence in daily activities $(\mathrm{BI}<49)$ having a prevalence of $71.5 \%$ compared to $28.4 \%$ in those without infections. Considering the different years of REPOSI, a substantial increase in the overall prevalence of patients with infections was observed, from $34.0 \%$ in the 2008 up to $52.1 \%$ in the 2010 , with an almost stable prevalence during the following years (with a mean value of $54.5 \%$ ) ( $P$ values $<0.0001$ for the overall chi squared comparison and 0.24 for the comparison after excluding the 2008 REPOSI run).

Before admission 187 patients $(6.5 \%)$ with infections lived in nursing homes, those from nursing homes being 4.7\% less among patients without infections. The greatest number of hospitalizations took place during the winter season, but a slightly higher prevalence of patients with infections was observed during summer (18.2\% vs $14.7 \%$ in those without infections).

The most common type of infections were in the upper and lower respiratory tract (38.2\%), of which 860 were pneumonia and bronchopneumonia, followed by those in the kidney and urinary tract $(21.1 \%)$ and the hepatobiliary system (9.3\%). Bloodstream infections accounted for the $5.5 \%$ of the infections (Table 2). An infection was the reason of hospitalization in 734 patients $(24.5 \%, 95 \%$ CI $23.0-26.1 \%)$, about half of them being hospitalized for respiratory tract infections, urinary tract and gastrointestinal infections accounting for $35.0 \%$ of the remaining infections. Respiratory tract infections were also the most common infection occurring during hospital stay, followed by those of the urinary tract. When analysing the type of infection over time, there was an almost stable pattern, with an increase only for septicemia (Supplementary Fig. 1). When we analysed infection sites according to sex, urinary (25.5\% vs $16.24 \%)$ and gastrointestinal $(8.5 \%$ vs $7.2 \%)$ infections occurred more frequently in females, hepatobiliary infections in males ( $10.6 \%$ vs $8.0 \%)$, and respiratory infections with no marked differences for sex distribution (35.3\% in females vs $41.4 \%$ in males). Respiratory tract $(35.9 \%$ vs $43.4 \%)$ and urinary tract infections (20.1\% vs $23.4 \%)$ were more prevalent among the oldest patients ( $\geq 85$ years), while hepatobiliary infections $(10.8 \%$ vs $5.9 \%$ ) were more prevalent in those less than 85 years of age.

Among patients with infections 176 (5.9\%) died during hospitalization.

All in all, approximately half of the older patients enrolled in the frame of the REPOSI register $(49.5 \%)$ had at least one infection diagnosed at admission or during hospital stay, and over the 8-year study period their prevalence remained almost unchanged after an early increase. In Italy, Orlando et al. have previously emphasized as an emerging problem that an increasing number of older people with multiple chronic conditions is admitted to hospital with infections, but actual data on older people acutely hospitalized in medical wards were scanty [9]. In the frame of the different runs of the REPOSI register, respiratory tract infections were the most frequent type of infection being more than one third of the cases $(38.2 \%)$, followed in prevalence by urinary tract infections throughout all the register runs. The same pattern was also observed by others [10]. Septicemia actually increased during the REPOSI runs from $11.3 \%$ in 2008 to $24.2 \%$ in 2016 . This can be explained by an increasing attention for this diagnosis and the large use of artificial devices in older patients.

The patients most frequently hospitalized in internal medicine wards are often very old and characterized by multiple chronic conditions, the most frequent concomitant diseases being diabetes and chronic renal failure. Polypharmacy is often a consequence of multimorbidity and indeed at admission more than half of our patients took 5 or more drugs. Other studies identified an association between infections and drug intake and it is known that some drugs frequently prescribed to old people increase the risk of infections [11]. In spite of the fact that a relatively small number of our older patients had a severe or total dependence according to the Barthel index (17.5\%), our data support the hypothesis that a compromised functional status and polypharmacy are associated with an increased prevalence of infections in the elderly [12]. In the previous studies, a poor functional status was found to be an independent prognostic factor for mortality in older people with pneumonia and a risk factor for respiratory and urinary tract infections [13, 14]. Furthermore, another REPOSI study found that a low Barthel index increased the risk of death in old patients admitted for pneumonia [15]. Finally, there was a lower degree of physical ability in patients with pneumonia developing during hospitalization compared to those admitted with community acquired pneumonia, as previous reported in a REPOSI study [6]. On the whole, the results of the present study, that considered all types of infections occurring in older people, suggest that a low functional status may be associated with all types of infection, not only with those of the respiratory tract. 
Table 1 Socio-demographic and clinical characteristics of 2991 older patients with infections and 3056 without infections at hospital admission

\begin{tabular}{|c|c|c|c|}
\hline Variables & $\begin{array}{l}\text { Patients with infec- } \\
\text { tion } N(\%)\end{array}$ & $\begin{array}{l}\text { Patients without } \\
\text { infections }\end{array}$ & Missing data \\
\hline 2008 & $453(15.2 \%)$ & $879(28.8 \%)$ & \\
\hline 2010 & $719(24.0 \%)$ & $661(21.6 \%)$ & \\
\hline 2012 & $703(23.5 \%)$ & $620(20.3 \%)$ & \\
\hline 2014 & $674(22.5 \%)$ & $538(17.6 \%)$ & \\
\hline 2016 & $442(14.8 \%)$ & $358(11.7 \%)$ & \\
\hline Women $(n, \%)$ & $1554(52.0 \%)$ & $1572(51.4 \%)$ & \\
\hline Age $($ mean $\pm S D)$ & $80 \pm 7.5$ & $78.6 \pm 7.4$ & \\
\hline \multicolumn{4}{|l|}{ Age group $(n, \%)$} \\
\hline Young old $(\leq 75)$ & $877(29.3 \%)$ & $1101(36.0 \%)$ & \\
\hline Old (76-84) & $1231(41.2 \%)$ & $1246(40.8 \%)$ & \\
\hline Oldest old $(\geq 85)$ & $883(29.5 \%)$ & $709(23.2 \%)$ & \\
\hline Living arrangement $(n, \%)$ & & & 219 \\
\hline Alone & $660(22.8 \%)$ & $657(22.4 \%)$ & \\
\hline With family member/caregiver & $2046(70.7 \%)$ & $2140(72.9 \%)$ & \\
\hline Nursing home & $187(6.5 \%)$ & $139(4.7 \%)$ & \\
\hline Barthel index score $(n, \%)$ & & & 1411 (1332 in 2008) \\
\hline Total dependence $(0-24)$ & $354(14.2 \%)$ & $124(5.8 \%)$ & \\
\hline Severe dependence $(25-49)$ & $229(9.2 \%)$ & $108(5.1 \%)$ & \\
\hline Moderate dependence (50-74) & $345(13.8 \%)$ & $241(11.3 \%)$ & \\
\hline Mild dependence (75-90) & $465(18.6 \%)$ & $403(18.9 \%)$ & \\
\hline Negligible dependence (91-100) & $1108(44.3 \%)$ & $1260(59.0 \%)$ & \\
\hline \multicolumn{4}{|l|}{ Number of drugs $(n, \%)$} \\
\hline 0 & $81(2.7 \%)$ & $113(3.7 \%)$ & \\
\hline $1-4$ & $1048(35.0 \%)$ & $1252(41.0 \%)$ & \\
\hline$\geq 5$ & $1862(62.3 \%)$ & $1691(55.3 \%)$ & \\
\hline \multicolumn{4}{|l|}{ Number of concomitant comorbidities } \\
\hline 0 & $25(0.8 \%)$ & $73(2.4 \%)$ & \\
\hline $1-2$ & $229(7.7 \%)$ & $351(11.5 \%)$ & \\
\hline $3-4$ & $455(15.2)$ & $674(22.0 \%)$ & \\
\hline$\geq 5$ & $2282(76.3 \%)$ & $1958(64.1 \%)$ & \\
\hline \multicolumn{4}{|l|}{ Season of hospitalization $(n, \%)$} \\
\hline Spring & $800(26.8 \%)$ & $827(27.1 \%)$ & \\
\hline Summer & $544(18.2 \%)$ & $450(14.7 \%)$ & \\
\hline Autumn & $707(23.6 \%)$ & $825(27 \%)$ & \\
\hline Winter & $940(31.4 \%)$ & $954(31.2 \%)$ & \\
\hline \multicolumn{4}{|l|}{ Associated chronic diseases $(n, \%)$} \\
\hline Diabetes & $844(28.2 \%)$ & $800(26.2 \%)$ & \\
\hline Heart failure & $504(16.8 \%)$ & $519(17.0 \%)$ & \\
\hline Dementia & $74(2.5 \%)$ & $488(16.0 \%)$ & \\
\hline Parkinson disease & $105(3.5 \%)$ & $428(14.0 \%)$ & \\
\hline Chronic renal failure & $567(19.0 \%)$ & $76(2.5 \%)$ & \\
\hline Cancer & $538(18.0 \%)$ & $38(1.2 \%)$ & \\
\hline Patient status at discharge $(n, \%)$ & & & 35 \\
\hline Home & $2556(85.8 \%)$ & $2695(88.9 \%)$ & \\
\hline Dead during hospitalization & $176(5.9 \%)$ & $86(2.8 \%)$ & \\
\hline Transferred to another medical ward & $249(8.4 \%)$ & $250(8.3 \%)$ & \\
\hline
\end{tabular}


Table 2 Prevalence of each infection typical in the REPOSI register from 2010 to 2016

\begin{tabular}{ll}
\hline Type of infection & Number (\%) \\
\hline Respiratory tract infections & $1357(38.2 \%)$ \\
Lower & 1074 \\
Upper & 267 \\
Not classified & 16 \\
Gastrointestinal tract infections & $281(7.9 \%)$ \\
Urinary tract infections & $750(21.1 \%)$ \\
Hepatobiliary infections & $330(9.3 \%)$ \\
Cardiac infections & $32(0.9 \%)$ \\
Septicemia & $194(5.5 \%)$ \\
Mycosis & $178(5.0 \%)$ \\
Others & $432(12.1 \%)$ \\
\hline
\end{tabular}

Others included: central nervous system infections, skin and soft tissue infections, osteomyelitis, infections due to internal prosthetic devices, implants and grafts, postoperative infections, tuberculosis and other mycobacteriosis, unspecified bacterial infections

There are limitations in this merely descriptive study, such as the fact that the register does not collect details on etiology, outcomes and diagnostic methods for infections (e.g. microbiological cultures, X-ray imaging, etc.). On the other hand REPOSI is based upon data from more than 100 internal medicine and geriatric wards throughout Italy, thus making this study representative of the hospitalized older population of this country. Moreover, data collection started in 2008, so that changes over time were accounted for.

In conclusion infections are frequent events diagnosed in older hospitalized patients with a high number of comorbidities and on polypharmacy, respiratory tract infections being the most frequent followed by urinary tract infections.

Acknowledgements REPOSI Investigators members: Domenico Prisco, Elena Silvestri, Giacomo Emmi, Alessandra Bettiol, Cenci Caterina (Azienda Ospedaliero Universitaria Careggi Firenze, Medicina Interna Interdisciplinare); Gianni Biolo, Michela Zanetti, Martina Guadagni, Michele Zaccari, Massimiliano Chiuch, Michele Zaccari (Azienda Sanitaria Universitaria Integrata di Trieste, Clinica Medica Generale e Terapia Medica); Massimo Vanoli, Giulia Grignani, Edoardo Alessandro Pulixi (Azienda Ospedaliera della Provincia di Lecco, Ospedale di Merate, Lecco, Medicina Interna); Mauro Bernardi, Silvia Li Bassi, Luca Santi, Giacomo Zaccherini (Azienda Ospedaliera Policlinico Sant'Orsola-Malpighi, Bologna, Semeiotica Medica Bernardi); Graziana Lupattelli, Elmo Mannarino, Vanessa Bianconi, Francesco Paciullo, Riccardo Alcidi (Azienda Ospedaliera Santa Maria della Misericordia, Perugia, Medicina Interna); Ranuccio Nuti, Roberto Valenti, Martina Ruvio, Silvia Cappelli, Alberto Palazzuoli (Azienda Ospedaliera Università Senese, Siena, Medicina Interna I); Domenico Girelli, Fabiana Busti, Giacomo Marchi (Azienda Ospedaliera Universitaria Integrata di Verona, Verona, Medicina Generale e Malattie Aterotrombotiche e Degenerative); Mario Barbagallo, Ligia Dominguez, Floriana Cocita, Vincenza Beneduce, Lidia Plances (Azienda Ospedaliera Universitaria Policlinico Giaccone Policlinico di Palermo, Palermo, Unità Operativa di Geriatria e Lungodegenza);
Salvatore Corrao, Giuseppe Natoli, Salvatore Mularo, Massimo Raspanti, Federica Cavallaro (A.R.N.A.S. Civico, Di Cristina, Benfratelli, Palermo, UOC Medicina Interna ad Indirizzo Geriatrico-Riabilitativo); Marco Zoli, Ilaria Lazzari, Mattia Brunori, Elisa Fabbri, Donatella Magalotti, Raffaella Arnò (Azienda Ospedaliera Universitaria Policlinico S. Orsola-Malpighi, Bologna, Unità Operativa di Medicina Interna); Franco Laghi Pasini, Pier Leopoldo Capecchi, (Azienda Ospedaliera Universitaria Senese, Siena, Unità Operativa Complessa Medicina 2); Giuseppe Palasciano, Maria Ester Modeo, Carla Di Gennaro (Azienda Ospedaliero-Universitaria Consorziale Policlinico di Bari, Bari, Medicina Interna Ospedaliera "L. D’Agostino", Medicina Interna Universitaria “A. Murri”); Maria Domenica Cappellini, Diletta Maira, Valeria Di Stefano, Giovanna Fabio, Sonia Seghezzi, Marta Mancarella, Margherita Migone De Amicis, Giacomo De Luca, Natalia Scaramellini (Fondazione IRCCS Cà Granda Ospedale Maggiore Policlinico, Milano, Unità Operativa Medicina Interna IA); Matteo Cesari, Paolo Dionigi Rossi, Sarah Damanti, Marta Clerici, Federica Conti, Giulia Bonini, Barbara Brignolo Ottolini (Fondazione IRCCS Cà Granda Ospedale Maggiore Policlinico, Milano, Geriatria); Antonio Di Sabatino, Emanuela Miceli, Marco Vincenzo Lenti, Martina Pisati, Costanza Caccia Dominioni (IRCCS Policlinico San Matteo di Pavia, Pavia, Clinica Medica I, Reparto 11); Giovanni Murialdo, Alessio Marra, Federico Cattaneo, Roberto Pontremoli, Valentina Beccati, Giulia Nobili (IRCCS Azienda Ospedaliera Universitaria San MartinoIST di Genova, Genova, Clinica di Medicina Interna 2); Maria Beatrice Secchi, Davide Ghelfi (Ospedale Bassini di Cinisello Balsamo, Milano, Divisione Medicina); Luigi Anastasio, Lucia Sofia, Maria Carbone (Ospedale Civile Jazzolino di Vibo Valentia, Vibo Valentia, Medicina interna); Francesco Cipollone, Maria Teresa Guagnano, Emanuele Valeriani, Ilaria Rossi (Ospedale Clinicizzato SS. Annunziata, Chieti, Clinica Medica); Gerardo Mancuso, Daniela Calipari, Mosè Bartone (Ospedale Giovanni Paolo II Lamezia Terme, Catanzaro, Unità Operativa Complessa Medicina Interna); Giuseppe Delitala, Maria Berria, Chiara Pes, Alessandro Delitala (Azienda ospedaliera-universitaria di Sassari, Clinica Medica); Maurizio Muscaritoli, Alessio Molfino, Enrico Petrillo (Policlinico Umberto I, Sapienza Università di Roma, Medicina Interna e Nutrizione Clinica Policlinico Umberto I); Giuseppe Zuccalà, Gabriella D'Aurizio (Policlinico Universitario A. Gemelli, Roma, Roma, Unità Operativa Complessa Medicina d'Urgenza e Pronto Soccorso) Giuseppe Romanelli, Alessandra Marengoni, Alberto Zucchelli, Francesca Manzoni, Andrea Volpini (Spedali Civili di Brescia, Brescia, Geriatria); Antonio Picardi, Umberto Vespasiani Gentilucci, Paolo Gallo, Chiara Dell’Unto (Università Campus Bio-Medico, Roma, Medicina Clinica-Epatologia); Giorgio Annoni, Maurizio Corsi, Giuseppe Bellelli, Sara Zazzetta, Paolo Mazzola, Hajnalka Szabo, Alessandra Bonfanti (Università degli studi di MilanoBicocca Ospedale S. Gerardo, Monza, Unità Operativa di Geriatria); Franco Arturi, Elena Succurro, Mariangela Rubino, Bruno Tassone, Giorgio Sesti (Università degli Studi Magna Grecia, Policlinico Mater Domini, Catanzaro, Unità Operativa Complessa di Medicina Interna); Maria Grazia Serra, Maria Antonietta Bleve (Azienda Ospedaliera "Cardinale Panico" Tricase, Lecce, Unità Operativa Complessa Medicina); Laura Gasbarrone, Maria Rosaria Sajeva (Azienda Ospedaliera Ospedale San Camillo Forlanini, Roma, Medicina Interna 1); Antonio Brucato, Silvia Ghidoni (Azienda Ospedaliera Papa Giovanni XXIII, Bergamo, Medicina 1); Fabrizio Fabris, Irene Bertozzi, Giulia Bogoni, Maria Victoria Rabuini, Elisabetta Cosi, Paolo Scarinzi, Annalisa Amabile, Elisabetta Omenetto, Tancredi Prandini (Azienda Ospedaliera Università di Padova, Padova, Clinica Medica I); Roberto Manfredini, Fabio Fabbian, Benedetta Boari, Alfredo De Giorgi, Ruana Tiseo, Roberto De Giorgio (Azienda Ospedaliera - Universitaria Sant'Anna, Ferrara, Unità Operativa Clinica Medica); Giuseppe Paolisso, Maria Rosaria Rizzo (Azienda Ospedaliera Universitaria della Seconda Università degli Studi di Napoli, Napoli, VI Divisione di Medicina Interna e Malattie Nutrizionali dell'Invecchiamento); Claudio Borghi, Enrico Strocchi, Eugenia Ianniello, Mario Soldati 
(Azienda Ospedaliera Universitaria Policlinico S. Orsola-Malpighi, Bologna, Unità Operativa di Medicina Interna Borghi); Carlo Sabbà, Francesco Saverio Vella, Patrizia Suppressa, Pasquale Agosti, Andrea Schilardi, Francesca Loparco, Giovanni Michele De Vincenzo, Alessio Comitangelo, Emanuele Amoruso (Azienda Ospedaliero-Universitaria Consorziale Policlinico di Bari, Bari, Medicina Interna Universitaria C. Frugoni); Luigi Fenoglio, Andrea Falcetta, Christian Bracco (Azienda Sanitaria Ospedaliera Santa Croce e Carle di Cuneo, Cuneo, S. C. Medicina Interna); Anna L. Fracanzani Silvia Fargion, , Silvia Tiraboschi, Annalisa Cespiati, Giovanna Oberti, Giordano Sigon (Fondazione IRCCS Cà Granda Ospedale Maggiore Policlinico, Milano, Medicina Interna 1B); Flora Peyvandi, Raffaella Rossio, Barbara Ferrari, Giulia Colombo (Fondazione IRCCS Cà Granda Ospedale Maggiore Policlinico, Milano, UOC Medicina generale - Emostasi e trombosi); Valter Monzani, Valeria Savojardo, Christian Folli, Giuliana Ceriani (Fondazione IRCCS Cà Granda Ospedale Maggiore Policlinico, Milano, Medicina Interna Alta Intensità); Francesco Salerno, Giada Pallini (IRCCS Policlinico San Donato e Università di Milano, San Donato Milanese, Medicina Interna); Franco Dallegri, Luciano Ottonello, Luca Liberale, Lara Caserza, Kassem Salam (Università di Genova, Genova, Medicina Interna 1); Nicola Lucio Liberato, Tiziana Tognin (ASST di Pavia, UOSD Medicina Interna, Ospedale di Casorate Primo, Pavia); Giovanni Battista Bianchi, Sabrina Giaquinto (Ospedale "SS Gerosa e Capitanio" di Lovere, Bergamo, Unità Operativa Complessa di Medicina Generale, Azienda Ospedaliera "Bolognini" di Seriate, Bergamo); Francesco Purrello, Antonino Di Pino, Salvatore Piro (Ospedale Garibaldi Nesima, Catania, Unità Operativa Complessa di Medicina Interna); Renzo Rozzini, Lina Falanga, Elena Spazzini, Camillo Ferrandina (Ospedale Poliambulanza, Brescia, Medicina Interna e Geriatria); Giuseppe Montrucchio, Paolo Petitti, Paolo Peasso, Edoardo Favale, Cesare Poletto (Dipartimento di Scienze Mediche, Università di Torino, Città della Scienza e della Salute, Torino, Medicina Interna 2 U. Indirizzo d'Urgenza); Raffaella Salmi, Piergiorgio Gaudenzi (Azienda Ospedaliera-Universitaria S. Anna, Ferrara, Unità Operativa di Medicina Ospedaliera II); Francesco Violi, Ludovica Perri (Policlinico Umberto I, Roma, Prima Clinica Medica); Raffaele Landolfi, Massimo Montalto, Antonio Mirijello (Policlinico Universitario A. Gemelli, Roma, Clinica Medica); Luigina Guasti, Luana Castiglioni, Andrea Maresca, Alessandro Squizzato, Leonardo Campiotti, Alessandra Grossi (Università degli Studi dell'Insubria, Ospedale di Circolo e Fondazione Macchi, Varese, Medicina Interna I); Marco Bertolotti, Chiara Mussi, Giulia Lancellotti, Maria Vittoria Libbra, Giulia Dondi, Elisa Pellegrini, Lucia Carulli, Matteo Galassi, Yasmine Grassi (Università di Modena e Reggio Emilia, Azienda Ospedaliero-Universitaria di Modena; Ospedale Civile di Baggiovara, Unità Operativa di Geriatria); Francesco Perticone, Maria Perticone, Rosa Battaglia, Marco FIlice, Raffaele Maio (Università Magna Grecia Policlinico Mater Domini, Catanzaro, Unità Operativa Malattie Cardiovascolari Geriatriche); Vincenzo Stanghellini, Eugenio Ruggeri, Sara del Vecchio (Dipartimento di Scienze Mediche e Chirurgiche, Unità Operativa di Medicina Interna, Università degli Studi di Bologna/ Azienda Ospedaliero-Universitaria S.Orsola-Malpighi, Bologna); Andrea Salvi, Roberto Leonardi, Giampaolo Damiani (Spedali Civili di Brescia, U.O. 3a Medicina Generale); William Capeci, Armando Gabrielli, Massimo Mattioli, Giuseppe Pio Martino, Lorenzo Biondi, Pietro Pettinari (Clinica Medica, Azienda Ospedaliera Universitaria Ospedali Riuniti di Ancona); Riccardo Ghio, Anna Dal Col (Azienda Ospedaliera Università San Martino, Genova, Medicina III); Salvatore Minisola, Luciano Colangelo, Mirella Cilli, Giancarlo Labbadia (Policlinico Umberto I, Roma, SMSC03 - Medicina Interna A e Malattie Metaboliche dell'osso); Antonella Afeltra, Benedetta Marigliano, Maria Elena Pipita (Policlinico Campus Biomedico Roma, Roma, Medicina Clinica); Pietro Castellino, Luca Zanoli, Samuele Pignataro, Alfio Gennaro, Julien Blanco (Azienda Ospedaliera Universitaria Policlinico - V. Emanuele, Catania, Dipartimento di Medicina); Valter Saracco, Marisa Fogliati, CarloBussolino (Ospedale Cardinal Massaia
Asti, Medicina A); Francesca Mete, Miriam Gino (Ospedale degli Infermi di Rivoli, Torino, Medicina Interna) Antonio Cittadini, Carlo Vigorito, Michele Arcopinto, Andrea Salzano, Emanuele Bobbio, Alberto Maria Marra, Domenico Sirico (Azienda Policlinico Universitario Federico II di Napoli, Napoli, Medicina Interna e Riabilitazione Cardiologica); Guido Moreo, Francesca Gasparini, Silvia Prolo, Gloria Pina (Clinica San Carlo Casa di Cura Polispecialistica, Paderno Dugnano, Milano, Unità Operativa di Medicina Interna); Alberto Ballestrero, Fabio Ferrando (Clinica Di Medicina Interna ad Indirizzo Oncologico, Azienda Ospedaliera Università San Martino di Genova); Sergio Berra, Simonetta Dassi, Maria Cristina Nava (Medicina Interna, Azienda Ospedaliera Guido Salvini, Garnagnate, Milano); Bruno Graziella, Stefano Baldassarre, Salvatore Fragapani, Gabriella Gruden (Medicina Interna III, Ospedale S. Giovanni Battista Molinette, Torino); Giorgio Galanti, Gabriele Mascherini, Cristian Petri, Laura Stefani (Agenzia di Medicina dello Sport, AOUC Careggi, Firenze); Margherita Girino, Valeria Piccinelli (Medicina Interna, Ospedale S. Spirito Casale Monferrato, Alessandria); Francesco Nasso, Vincenza Gioffrè, Maria Pasquale (Struttura Operativa Complessa di Medicina Interna, Ospedale Santa Maria degli Ungheresi, Reggio Calabria); Giuseppe Scattolin, Sergio Martinelli, Mauro Turrin (Medicina Interna, Ospedale di Monselice, Padova); Leonardo Sechi, Cristina Catena, Gianluca Colussi (Clinica Medica, Azienda Ospedaliera Universitaria, Udine). Nicola Passariello, Luca Rinaldi (Presidio Medico di Marcianise, Napoli, Medicina Interna); Franco Berti, Giuseppe Famularo, Patrizia Tarsitani (Azienda Ospedaliera San Camillo Forlanini, Roma, Medicina Interna II); Roberto Castello, Michela Pasino (Ospedale Civile Maggiore Borgo Trento, Verona, Medicina Generale e Sezione di Decisione Clinica); Gian Paolo Ceda, Marcello Giuseppe Maggio, Simonetta Morganti, Andrea Artoni (Azienda Ospedaliero Universitaria di Parma, U.O.C Clinica Geriatrica); Stefano Del Giacco, Davide Firinu, Francesca Losa, Giovanni Paoletti, Giulia Costanzo (Policlinico Universitario Duilio Casula, Azienda Ospedaliero-Universitaria di Cagliari, Cagliari, Medicina Interna, Allergologia ed Immunologia Clinica); Giuseppe Montalto, Anna Licata, Valentina Malerba, Filippo Alessandro Montalto (Azienda Ospedaliera Universitaria Policlinico Paolo Giaccone, Palermo, UOC di Medicina Interna); Antonino Lasco, Giorgio Basile, Antonino Catalano (Azienda Ospedaliera Universitaria Policlinico G. Martino, Messina, Unità Operativa di Geriatria); Lorenzo Malatino, Benedetta Stancanelli, Valentina Terranova, Salvatore Di Marca, Rosario Di Quattro, Lara La Malfa, Rossella Caruso (Azienda Ospedaliera per l'Emergenza Cannizzaro, Catania, Clinica Medica Università di Catania); Patrizia Mecocci, Carmelinda Ruggiero, Virginia Boccardi (Università degli Studi di Perugia-Azienda Ospedaliera S.M. della Misericordia, Perugia, Struttura Complessa di Geriatria); Tiziana Meschi, Fulvio Lauretani, Andrea Ticinesi, Antonio Nouvenne (Azienda Ospedaliera Universitaria di Parma, U.O Medicina Interna e Lungodegenza Critica); Pietro Minuz, Luigi Fondrieschi (Azienda Ospedaliera Universitaria Verona, Policlinico GB Rossi, Verona, Medicina Generale per lo Studio ed il Trattamento dell'Ipertensione Arteriosa); Mario Pirisi, Gian Paolo Fra, Daniele Sola (Azienda Ospedaliera Universitaria Maggiore della Carità, Medicina Interna 1); Massimo Porta, Piero Riva (Azienda Ospedaliera Universitaria Città della Salute e della Scienza di Torino, Medicina Interna 1U); Roberto Quadri, Erica Larovere, Marco Novelli (Ospedale di Ciriè, ASL TO4, Torino, S.C. Medicina Interna); Giorgio Scanzi, Caterina Mengoli, Stella Provini, Laura Ricevuti (ASST Lodi, Presidio di Codogno, Milano, Medicina); Emilio Simeone, Rosa Scurti, Fabio Tolloso (Ospedale Spirito Santo di Pescara, Geriatria); Roberto Tarquini, Alice Valoriani, Silvia Dolenti, Giulia Vannini (Ospedale San Giuseppe, Empoli, USL Toscana Centro, Firenze, Medicina Interna I); Alberto Tedeschi, Lucia Trotta (ASST Fatebenefratelli - Sacco, Milano, Medicina Interna a indirizzo Pneumologico); Riccardo Volpi, Pietro Bocchi, Alessandro Vignali (Azienda Ospedaliera Universitaria di Parma, Clinica e Terapia Medica). Sergio Harari, Chiara Lonati, Mara 
Cattaneo, Federico Napoli (Ospedale San Giuseppe Multimedica Spa, U.O. Medicina Generale).

Funding None.

\section{Compliance with ethical standards}

Conflict of interest FP has received honoraria for participating as a speaker at satellite symposia and educational meetings organized by Ablynx, Grifols, Novo Nordisk, Roche, Shire and Sobi. She has received consulting fees from Kedrion and she is member of the scientific advisory board of Ablynx. Other authors have no conflict of interest to declare.

Statements on human and animal rights REPOSI was accepted by the ethical committees of all participant hospitals.

Informed consent Each patient supplied informed consent.

\section{References}

1. Liang SY (2016) Sepsis and other infectious disease emergencies in the elderly. Emerg Med Clin N Am 34:501-522

2. Shi T, Denouel A, Tietjen AK, Lee JW, Falsey AR, Demont C, Nyawanda BO, Cai B, Fuentes R, Stoszek SK, Openshaw P, Campbell H, Nair H (2019) Global and regional burden of hospital admissions for pneumonia in older adults: a systematic review and meta-analysis. J Infect Dis. https://doi.org/10.1093/infdis/jiz053

3. Kardas P, Ratajczyk-Pakalska E (2003) Reasons for elderly patient hospitalization in departments of internal medicine in Lodz. Aging Clin Exp Res 15:25-31

4. Falcone M, Paul M, Yahav D, Orlando G, Tiseo G, Prendki V, Güerri-Fernández R, Gavazzi G, Mutters NT, Cookson B, Tinelli M (2019) Study Group for Infections in the elderly (ESGIE) antimicrobial consumption and impact of antimicrobial stewardship programmes in long-term care facilities. Clin Microbiol Infect 25:562-569

5. Ardoino I, Mannucci PM, Nobili A, Franchi C (2019) Antibiotic use and associated factors in a large sample of hospitalized older people. J Glob Antimicrob Resist. https://doi.org/10.1016/j. jgar.2019.04.013

6. Rossio R, Franchi C, Ardoino I, Djade CD, Tettamanti M, Pasina L, Salerno F, Marengoni A, Corrao S, Marcucci M, Peyvandi F, Biganzoli EM, Nobili A, Mannucci PM (2015) Adherence to antibiotic treatment guidelines and outcomes in the hospitalized elderly with different types of pneumonia. Eur J Intern Med 26:330-337

7. Mahoney FI, Barthel DW (1965) Functional evaluation: the Barthel Index. Md State Med J 14:61-65

8. Christensen KL, Holman RC, Steiner CA, Sejvar JJ, Stoll BJ, Schonberger LB (2009) Infectious disease hospitalizations in the United States. Clin Infect Dis 49:1025-1035

9. Orlando G, Gubertini G, Negri C, Coen M, Ricci E, Galli M, Rizzardini G (2014) Trends in hospital admissions at a department for infectious diseases in Italy from 1995 to 2011 and implications for health policies. Public Health 14:980

10. Kucukardali Y, Oncul O, Kunter E, Turhan V, Solmazgul E, Terekeci HM, Sayan O, Oktenli C (2009) Community acquired infections in elderly population Cent. Eur J Med 4:171-178

11. Maes ML, Fixen DR, Linnebur SA (2017) Adverse effects of proton-pump inhibitor use in older adults: a review of the evidence. Ther Adv Drug Saf 8:273-297

12. High K, Bradley S, Loeb M, Palmer R, Quagliarello V, Yoshikawa $T$ (2005) A new paradigm for clinical investigation of infectious syndrome in older adults: assessing functional status as a risk factor and outcome measure. J Am Geriatri Soc 53:528-535

13. Osakwe ZT, Larson E, Shang J (2019) Urinary tract infection related hospitalization among older adults receiving home health care. Am J Infect Control 47:786-792

14. Mody L, Sun R, Bradley S (2006) Assessment of pneumonia in older adults: effect of functional status. J Am Geriatr Soc 54:1062-1067

15. Corrao S, Argano C, Natoli G, Nobili A, Corazza GR, Mannucci PM, Perticone F (2018) Disability, and not diabetes, is a strong predictor of mortality in oldest old patients hospitalized with pneumonia. Eur J Intern Med 54:53-59

Publisher's Note Springer Nature remains neutral with regard to jurisdictional claims in published maps and institutional affiliations. 
Internal \& Emergency Medicine is a copyright of Springer, 2019. All Rights Reserved. 